\title{
Repeated Exposure to Dissection Does Not Influence Students' Attitudes towards Human Body Donation for Anatomy Teaching
}

\author{
Philip Maseghe Mwachaka, Pamela Mandela, and Hassan Saidi \\ Department of Human Anatomy, College of Health Sciences, School of Medicine, University of Nairobi, P.O. Box 30197, \\ Nairobi 00100, Kenya \\ Correspondence should be addressed to Philip Maseghe Mwachaka; pmaseghe@gmail.com
}

Received 19 January 2016; Revised 5 March 2016; Accepted 29 March 2016

Academic Editor: Friedrich Paul Paulsen

Copyright ( 2016 Philip Maseghe Mwachaka et al. This is an open access article distributed under the Creative Commons Attribution License, which permits unrestricted use, distribution, and reproduction in any medium, provided the original work is properly cited.

\begin{abstract}
The use of unclaimed bodies for anatomical dissection has been the main method of instruction at our institution. There is however a shortage of cadavers for dissection given the increase in the number of medical schools as well as in the number of students enrolling in these schools. This shortage could be mitigated by having voluntary human body donation programs. This study aimed at assessing the attitudes of medical students and surgical residents towards body donation for anatomy learning. We conducted an online survey involving 72 first-year medical students and 41 surgical residents at University of Nairobi who had completed one year of anatomy dissection. For the medical students, this was their first dissection experience while it was the second exposure for the surgery trainees. Most of the surgical trainees (70.7\%) and medical students (68.1\%) were opposed to self-body donation. This was mainly due to cultural (37\%) and religious (20\%) barriers. Surprisingly, of those not willing to donate themselves, $67.9 \%$ (82.8\% surgical trainees, $59.2 \%$ medical students) would recommend the practice to other people. Exposure to repeated dissection does not change the perceptions towards body donation. It is noteworthy that culture and religion rank high as clear barriers amongst this "highly informed" group of potential donors.
\end{abstract}

\section{Introduction}

Human cadavers are important in the initial and continuing training of medical doctors and advancement of medical research [1-3]. Cadaveric dissection has been the main mode of learning anatomy for many medical schools $[4,5]$. Whereas donated cadavers make up $80-100 \%$ of the total cadavers in European and North American medical schools, up to $90 \%$ of African medical schools still rely on unclaimed bodies [6-9]. In Kenya, all medical schools use unclaimed bodies obtained from public health facilities around the country [10]. At the University of Nairobi, the oldest medical school in Kenya, all the cadavers used for dissection are unclaimed bodies $[10,11]$. This school runs a 6 -year undergraduate bachelor of medicine and surgery course and a postgraduate surgical residency course. Students in these two groups spend at least 250 hours of their first year of study dissecting the human body.

With the increasing demand for more healthcare workers in the country, many medical schools have been set up. The number of medical schools in Kenya has grown from one in 1967 to nine in 2015, with each school having massive expansion in student enrollment. All these schools compete for the same pool of unclaimed bodies resulting in shortage of cadavers for dissection. For instance, despite increased medical student enrollment at the University of Nairobi to over 400 students per year, the supply of cadavers has stagnated at 50 per year. This cadaver shortage has led to increase in the cadaver to student ratio from 1:6 to 1:9.

Scarcity of unclaimed bodies for dissection necessitates development of human body bequest programs $[9,12,13]$ especially in African countries which have solely been relying on unclaimed bodies for anatomy teaching $[7,10]$. Although the University of Nairobi, Kenya, has a human body donation program, it hardly receives any donated bodies. For instance, only two bodies have been donated in the last year. This study therefore aimed at assessing the attitudes of the end-users (the students) towards body donation and whether a repeat 
exposure to cadaveric dissection would positively influence the student perceptions towards body donation.

\section{Methods}

2.1. Participants and Setting. This study enrolled first-year medical (undergraduate) students and surgical (postgraduate) residents at the University of Nairobi (UoN) in Kenya, after they had completed one year of cadaveric dissection. For the medical students, this was their first dissection experience while it was the second exposure for the surgery trainees, having dissected when they were undergraduate medical students. After ethical approval by the School of Medicine, University of Nairobi, an anonymous online based questionnaire was sent to 150 undergraduate and 55 postgraduate students. All participants were informed of the aims of the study, and their involvement was voluntary. This survey was conducted between 11 April, 2015, and 10 May, 2015. Seventytwo undergraduate (response rate $48 \%$ ) and 41 postgraduate (response rate 74.5\%) students completed the survey.

2.2. Questionnaire. Variables collected in the self-administered questionnaire included the course, gender, whether they would donate their bodies for anatomy teaching (yes/no answer), reasons for/against body donation (open-ended answer), whether they have heard of any local body donation program (yes/no answer), whether they would recommend body donation to other people (yes/no answer), and the reasons for recommending so (open-ended answer).

2.3. Statistical Analysis. Statistical analysis was done using the Statistical Package for the Social Sciences (SPSS) for Windows version 21.0 (SPSS Inc., Chicago, IL). The Chi-square test was used to compare the responses between the undergraduate (medical) and postgraduate (surgical) students. A $p$ value $<0.05$ was considered significant.

\section{Results}

3.1. Awareness of a Local Body Donation Program. Of the 113 students surveyed, $24.8 \%$ had heard of the body donation program at the UoN. More of the postgraduate students (43.9\%) compared to medical students (13.9\%) were aware of the local body bequest program (Table 1). The Chi-square test posted a Pearson $\chi^{2}=12.626$ and $p<0.001$, indicating a highly statistically significant difference in the proportionate distribution of those who were aware of a local body donation program.

3.2. Willingness to Donate Their Bodies. Only 24 (21.2\%) of the respondents were willing to donate their own bodies (Figure 1). Only $16(22.2 \%)$ of the undergraduates and 8 (19.5\%) of the postgraduates were willing to donate their own bodies $(p=0.943)$. It is noteworthy that $11(9.7 \%)$ students were undecided on whether they would donate or not.

3.3. Reasons for Being Not Willing to Donate Their Bodies. Of those who were not willing to donate, 70 respondents gave their reasons which were mainly cultural $(37.1 \%)$ and religious (20.0\%) barriers (Table 2). Apart from cultural and

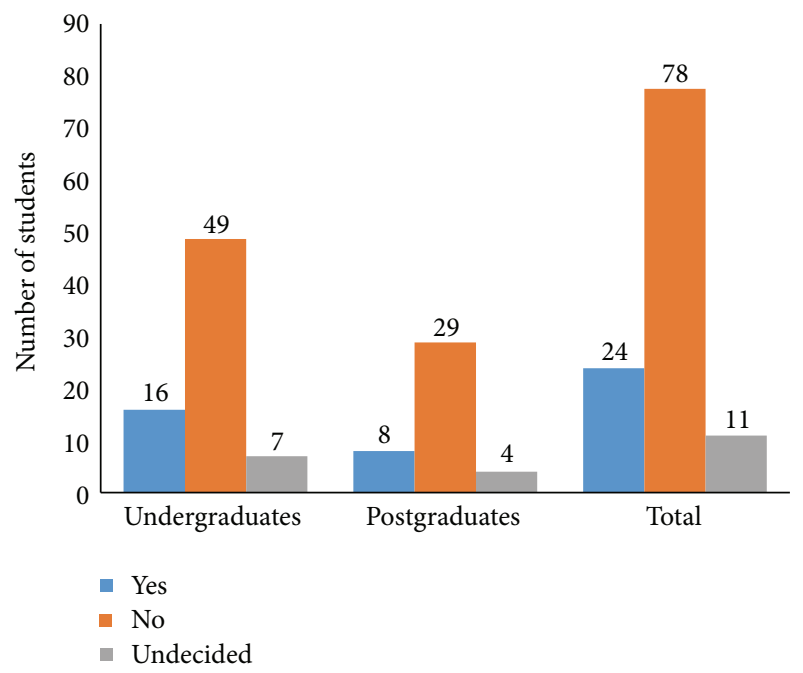

FIGURE 1: Proportions of students willing to donate their bodies for anatomy teaching.

TABLE 1: Proportions of students aware of a body donation program at UoN.

\begin{tabular}{lccc}
\hline $\begin{array}{l}\text { Have you heard of any } \\
\text { local body donation } \\
\text { program? }\end{array}$ & Undergraduate Postgraduate & Total \\
\hline Yes & $10(13.9 \%)$ & $18(43.9 \%)$ & $28(24.8 \%)$ \\
No & $62(86.1 \%)$ & $23(56.1 \%)$ & $85(75.2 \%)$ \\
Total & $72(100.0 \%)$ & $41(100.0 \%)$ & $113(100.0 \%)$ \\
\hline
\end{tabular}

TABLE 2: Reasons for being not willing to donate their bodies.

\begin{tabular}{lccc}
\hline Reasons & Undergraduate & Postgraduate & Total \\
\hline Cultural & $13(31.7 \%)$ & $13(44.8 \%)$ & $26(37.1 \%)$ \\
Religious & $7(17.1 \%)$ & $7(24.1 \%)$ & $14(20.0 \%)$ \\
Others & $21(51.2 \%)$ & $9(31.0 \%)$ & $30(42.9 \%)$ \\
Total & $41(100.0 \%)$ & $29(100.0 \%)$ & $70(100.0 \%)$ \\
\hline
\end{tabular}

religious reasons, $16(22.8 \%)$ of the undergraduates felt that the amount of mutilation done at cadaveric dissection was too much. They decried the handling of cadavers especially by fellow students, terming it as undignified, uncomfortable, brutal, and sad. Three students felt the human body is sacred and should be treated as such. Two students thought that their relatives would not find closure if they donated their bodies. The postgraduates had similar thoughts. Five (17.2\%) of these students decried the level of mutilation at the dissection laboratory and found the idea of students practicing on their bodies to be rather repugnant. Two of the students would consider body donation for professional development of surgical specialization skills training, arguing that this mature set of students would better appreciate the body donation than do undergraduates. Two postgraduates preferred organ donation to whole body donation as they felt that it saves lives.

3.4. Recommendation of Body Donation for Anatomy Teaching to Other People. There were 84 (74.3\%) of students who were 
TABLE 3: Proportions of students not willing to donate their bodies but who would recommend body donation for anatomy teaching to other people.

\begin{tabular}{lccc}
\hline $\begin{array}{l}\text { Recommend body } \\
\text { donation to other } \\
\text { people? }\end{array}$ & Undergraduate & Postgraduate & Total \\
\hline Yes & $29(59.2 \%)$ & $24(82.8 \%)$ & $53(67.9 \%)$ \\
No & $20(40.8 \%)$ & $5(17.2 \%)$ & $25(32.1 \%)$ \\
Total & $49(100.0 \%)$ & $29(100.0 \%)$ & $78(100.0 \%)$ \\
\hline
\end{tabular}

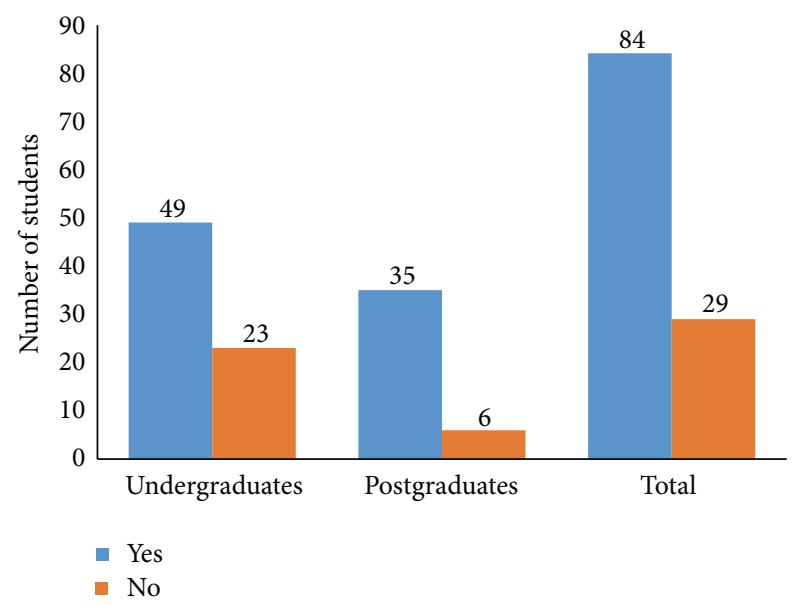

Figure 2: Recommendation of body donation to other people.

willing to recommend body donation for anatomy teaching to other people (Figure 2). Significantly more postgraduate, $35(85.4 \%)$, than undergraduate, 49 (68.1\%), students would recommend body donation for anatomy teaching to other people $(p=0.043)$. Surprisingly, $53(67.9 \%)$ respondents who were not willing to donate their own bodies would recommend body donation for teaching anatomy to other people (Table 3). Notably, more postgraduate students (82.8\%), who were not willing to donate their bodies, will recommend the practice to others in comparison with the undergraduate students $(59.2 \%)$ with a similar view $(p=0.031$; Table 3$)$.

3.5. Reasons for Recommending Body Donation to Other People. All the respondents who will recommend body donation to others noted that there was a need for more cadavers for teaching and research and this was the motivation for their recommendation. They also acknowledged cadaveric dissection as the best way to learn anatomy for future doctors. They however stated that potential donors should be provided with information on how the bodies will be handled before, during, and after dissection. In addition, the postgraduates recognized the need for body donation particularly for surgical skills training. They were also aware of the looming shortage of unclaimed bodies for cadaveric dissection.

\section{Discussion}

This study was seeking to determine the perception of undergraduate and postgraduate students towards body donation for learning anatomy. Body donation is an altruistic act which involves giving one's body after death for medical education and research [7]. The small proportion of students who are aware of a local body donation is an indicator that there is a lack of general information about the program. This could be due to lack of active campaigns about the importance of body donation for anatomy teaching. It is also possible that body donation as a source of cadavers for dissection may not have been emphasized to the students during induction to dissection classes, because all the cadavers used are from unclaimed bodies. This lack of knowledge of body donation programs is a barrier to becoming active donors [14-16]. Therefore, there is a need to educate both the students and the general public on the need and availability of body bequest programs $[12,17-20]$. The students need to be involved right from the time they start their studies [9].

Unwillingness to enroll in body bequest program by medical students and professionals seen in our study has also been reported in other studies. For instance, only 13.5\% of first-year medical students in France [21] and 6\% and $2 \%$ of medical students and doctors, respectively, in India were willing to donate their bodies for dissection [22]. A study on first-year Irish Medical students reported decreased support for body donation by the students from $31.5 \%$ before dissection to $19.6 \%$ after dissecting for 9 weeks [23]. A study in India reported that only $22 \%$ of physicians were willing to donate their bodies for medical education, but $68 \%$ expected the public to donate [17]. Surprisingly, even anatomists themselves are not willing to donate their bodies [17, 24]. In these two studies, only $15.7 \%$ of Turkish [17] and $25 \%$ of Dutch [24] anatomists were willing to donate their bodies.

The reasons against self-body donation given in the current study, namely, religious beliefs and customs as well as poor handling of the bodies during dissection, have been reported in other studies $[25,26]$. Studies in India have also reported firm religious beliefs and customs and the fear that the donated body will not be treated with respect and dignity as hindrances towards successful body donation programs [26]. Australian chiropractic students reported that atheistic and agnostic students were more willing to donate than religious students [25]. The same study also reported that willingness to donate one's own or a family member's body decreased as year of study increases, suggesting a possible negative impact of exposure to cadavers in the anatomy laboratory [25]. Other studies on the characteristics of body donors have also revealed that most people who registered as donors have no religious affiliations $[13,27,28]$.

For a body bequest program to be successful, issues raised by the students need to be addressed. Clear guidelines need to be laid down on the handling of the cadavers from the time they are received, during dissections, and in final disposal of the remnants of dissections $[29,30]$. Cadavers should be handled with respect and honor throughout the dissection period. Respectable gestures such as referring to the cadaver as the "silent teacher" rather than just an anatomy specimen have been shown to have a positive influence on the attitudes of medical students towards the cadaver [7, 31]. We should also borrow from other body bequest programs that have a dedication service before start of dissection and a 
thanksgiving or memorial service at the end of the dissection period [9, 29, 32, 33]. During the dedication services, the cadavers are dedicated to the training of the students, and the students are taught to value and respect the cadavers [9]. At the end of each dissection year, the general public, students, and staff come together to bid farewell to the donors in a decent burial ceremony $[9,29,32,33]$. This helps reassure apprehensive prospective donors that their remains will be treated with dignity.

\section{Conclusion}

This study has demonstrated that whereas students expect to learn anatomy by dissecting, as a potential donor population, they are reluctant to become donors themselves. Exposure to repeated dissection does not have a positive influence on the perceptions towards body donation. Most are hindered by cultural and religious reasons from participating in the body donation program. Lack of awareness of local bequest program may be a major hindrance to body donation.

\section{Competing Interests}

The authors declare that they have no competing interests.

\section{References}

[1] L.-A. Arráez-Aybar, I. Sánchez-Montesinos, R.-M. Mirapeix, B. Mompeo-Corredera, and J.-R. Sañudo-Tejero, "Relevance of human anatomy in daily clinical practice," Annals of Anatomy, vol. 192, no. 6, pp. 341-348, 2010.

[2] M. Anteby, F. Garip, P. V. Martorana, and S. Lozanoff, "Individuals' decision to co-donate or donate alone: an archival study of married whole body donors in Hawaii," PLoS ONE, vol. 7, no. 8, Article ID e42673, 2012.

[3] E. Brenner and D. Pais, "The philosophy and ethics of anatomy teaching," European Journal of Anatomy, vol. 18, no. 4, pp. 353360, 2014.

[4] J. C. McLachlan, "New path for teaching anatomy: living anatomy and medical imaging vs. dissection," Anatomical Record. Part B New Anatomist, vol. 281, no. 1, pp. 4-5, 2004.

[5] J. C. McLachlan and D. Patten, "Anatomy teaching: ghosts of the past, present and future," Medical Education, vol. 40, no. 3, pp. 243-253, 2006.

[6] H. Gangata, P. Ntaba, P. Akol, and G. Louw, "The reliance on unclaimed cadavers for anatomical teaching by medical schools in Africa," Anatomical Sciences Education, vol. 3, no. 4, pp. 174$183,2010$.

[7] E. J. Mazyala, M. Revocatus, M. Manyama et al., "Human bodies bequest program: a wake-up call to Tanzanian medical schools," Advances in Anatomy, vol. 2014, Article ID 940472, 6 pages, 2014.

[8] B. M. Riederer, "Body donations today and tomorrow: what is best practice and why?" Clinical Anatomy, vol. 29, no. 1, pp. 1118, 2016.

[9] S. N. Biasutto, N. Sharma, A. H. Weiglein et al., "Human bodies to teach anatomy: importance and procurement-experience with cadaver donation," Revista Argentina de Anatomía Clínica, vol. 6, no. 2, pp. 72-86, 2014.

[10] K. Ongeti, "Pedagogical value of dissection anatomy in Kenya," Singapore Medical Journal, vol. 53, no. 11, pp. 712-714, 2012.
[11] J. Ogeng'o, K. Ongeti, M. Misiani, and B. Olabu, "Maintaining excellence in the teaching of human anatomy: university of Nairobi experience," Anatomy Journal of Africa, vol. 2, no. 1, pp. 117-129, 2013.

[12] R. Ajita and Y. I. Singh, "Body donation and its relevance in anatomy learning-a review," Journal of the Anatomical Society of India, vol. 56, no. 1, pp. 44-47, 2007.

[13] L.-A. Arráez-Aybar, J. L. Bueno-López, and B. J. Moxham, "Anatomists' views on human body dissection and donation: an international survey," Annals of Anatomy, vol. 196, no. 6, pp. 376-386, 2014.

[14] L. E. Boulware, L. E. Ratner, L. A. Cooper, T. A. LaVeist, and N. R. Powe, "Whole body donation for medical science: a population-based study," Clinical Anatomy, vol. 17, no. 7, pp. 570-577, 2004.

[15] J. M. Marqués-Lespier, N. M. Ortiz-Vega, M. C. Sánchez, O. E. Soto-Avilés, and E. A. Torres, "Knowledge of and attitudes toward organ donation: a survey of medical students in Puerto Rico," Puerto Rico Health Sciences Journal, vol. 32, no. 4, pp. 187193, 2013.

[16] C. Green, D. Bowden, D. Molony, N. Burke, P. Felle, and S. Dudeney, "Attitudes of the medical profession to whole body and organ donation," The Surgeon-journal of The Royal Colleges of Surgeons of Edinburgh and Ireland, vol. 12, no. 2, pp. 73-77, 2014.

[17] Ü.S. Şehirli, E. Saka, and Ö. Sarikaya, "Attitudes of Turkish anatomists toward cadaver donation," Clinical Anatomy, vol. 17, no. 8, pp. 677-681, 2004.

[18] S. K. Chakraborty, H. Khan, S. Islam, and B. A. Yousuf, "Body donation and its significance in anatomy learning in Bangladesh-a review," Bangladesh Journal of Anatomy, vol. 8, no. 2, pp. 85-88, 2010.

[19] K. Ballala, A. Shetty, and S. B. Malpe, "Knowledge, attitude, and practices regarding whole body donation among medical professionals in a hospital in India," Anatomical Sciences Education, vol. 4, no. 3, pp. 142-150, 2011.

[20] S. J. Pundge, P. Wadekar, S. Gangane, and S. Borate, "Body donation and effect of awareness campaigns in Mumbai region," International Journal of Current Research and Academic Review, vol. 6, no. 20, pp. 70-73, 2014.

[21] D. Mekahli, A. Liutkus, S. Fargue, B. Ranchin, and P. Cochat, "Survey of first-year medical students to assess their knowledge and attitudes toward organ transplantation and donation," Transplantation Proceedings, vol. 41, no. 2, pp. 634-638, 2009.

[22] A. Saha, A. Sarkar, and S. Mandal, "Body donation after death: the mental setup of educated people," Journal of Clinical and Diagnostic Research, vol. 9, no. 6, pp. AC05-AC09, 2015.

[23] K. C. Cahill and R. R. Ettarh, "Student attitudes to whole body donation are influenced by dissection," Anatomical Sciences Education, vol. 1, no. 5, pp. 212-216, 2008.

[24] S. Bolt, E. Venbrux, R. Eisinga, and P. O. Gerrits, "Anatomist on the dissecting table? Dutch anatomical professionals' views on body donation," Clinical Anatomy, vol. 25, no. 2, pp. 168-175, 2012.

[25] M. Alexander, M. Marten, E. Stewart, S. Serafin, and G. Štrkalj, "Attitudes of Australian chiropractic students toward whole body donation: a cross-sectional study," Anatomical Sciences Education, vol. 7, no. 2, pp. 117-123, 2014.

[26] S. A. Rokade and A. P. Gaikawad, "Body donation in India: social awareness, willingness, and associated factors," Anatomical Sciences Education, vol. 5, no. 2, pp. 83-89, 2012. 
[27] J. Cornwall, G. F. Perry, G. Louw, and M. D. Stringer, "Who donates their body to science? An international, multicenter, prospective study," Anatomical Sciences Education, vol. 5, no. 4, pp. 208-216, 2012.

[28] G. Bajor, W. Likus, P. Kuszewski, K. Kostro, A. Łoś, and P. Kłakus, "'Mortui vivos docent' or who gives his body to science? The analysis of the personal questionnaires of Polish donors in the Conscious Body Donation Program," PLoS ONE, vol. 10, no. 3, Article ID e0121061, 2015.

[29] B. M. Riederer and J. L. Bueno-López, "Anatomy, respect for the body and body donation-a guide for good practice," European Journal of Anatomy, vol. 18, no. 4, pp. 361-368, 2014.

[30] B. M. Riederer, S. Bolt, E. Brenner et al., "The legal and ethical framework governing Body Donation in Europe-1st update on current practice," European Journal of Anatomy, vol. 16, no. 1, pp. 1-21, 2012.

[31] M. Bohl, P. Bosch, and S. Hildebrandt, "Medical students' perceptions of the body donor as a 'First Patient' or 'Teacher': a pilot study," Anatomical Sciences Education, vol. 4, no. 4, pp. 208-213, 2011.

[32] A. Winkelmann and F. H. Güldner, "Cadavers as teachers: the dissecting room experience in Thailand," British Medical Journal, vol. 329, no. 7480, pp. 1455-1457, 2004.

[33] J.-T. Park, Y. Jang, M. S. Park et al., "The trend of body donation for education based on Korean social and religious culture," Anatomical Sciences Education, vol. 4, no. 1, pp. 33-38, 2011. 

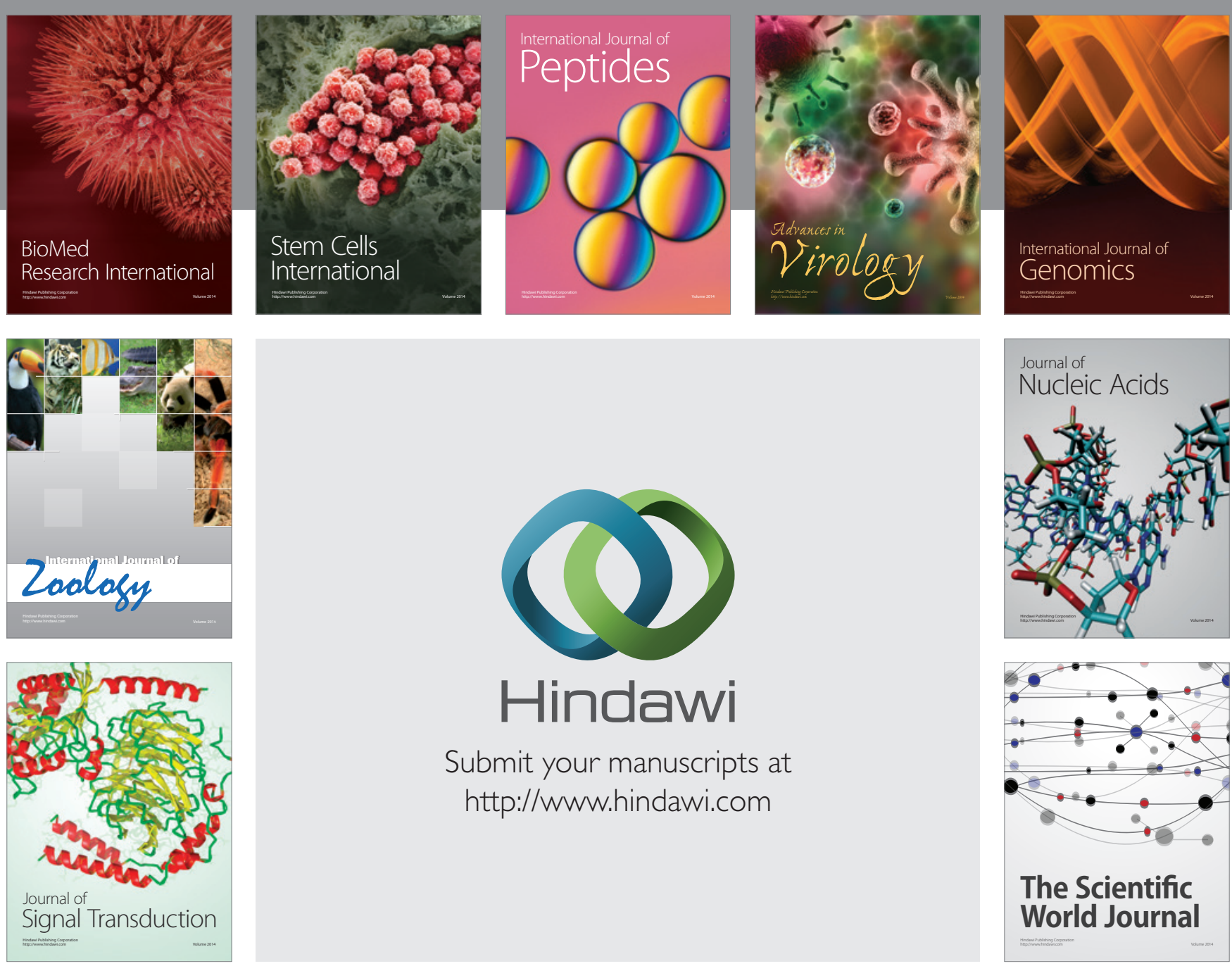

Submit your manuscripts at

http://www.hindawi.com
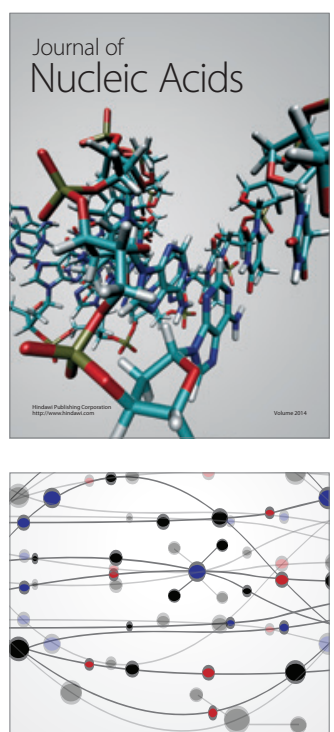

The Scientific World Journal
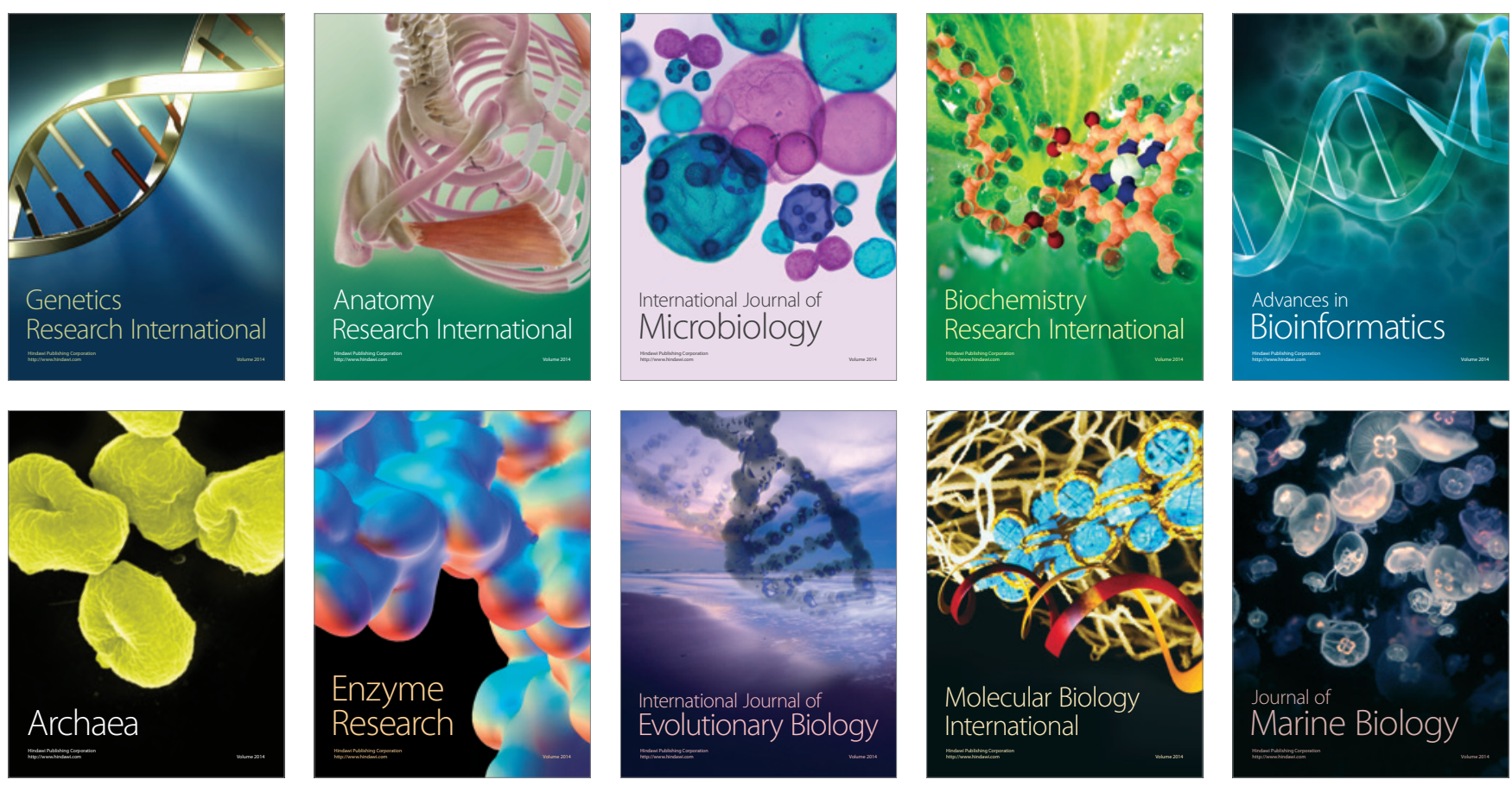